\title{
On the distribution of EGRET unidentified sources in the Galactic plane
}

\author{
D. Bhattacharya ${ }^{1,4}$, A. Akyüz ${ }^{2}$, T. Miyagi ${ }^{1}$, J. Samimi $^{3}$, and A. Zych ${ }^{1}$ \\ ${ }^{1}$ Institute of Geophysics and Planetary Physics, University of California, Riverside, CA 92521, USA \\ 2 Dept. of Physics, Cukurova University, Adana, Turkey \\ 3 Dept. pf Physics, Sharif University of Technology, Tehran, Iran \\ ${ }^{4}$ Riverside Community College, Riverside, CA 92506, USA
}

Received 16 September 2002 / Accepted 10 March 2003

\begin{abstract}
It has been suggested that young pulsars, with ages less than a million years, are possible counterparts of some of the Galactic unidentified gamma-ray sources detected by the EGRET instrument on-board the Compton Gamma Ray Observatory. In this work, we compare different aspects of the EGRET unidentified (EUI) source distribution in the Galactic plane with those of the pulsar distribution. An EUI source $\log N-\log S$ analysis is presented and compared with the Galactic radio pulsar $\log N-\log S$ distribution. A number of systematic effects that could introduce errors to the EGRET $\log N-\log S$ relation are discussed. A two-point angular correlation analysis of the EUI sources and Galactic pulsars is performed. We find that the global distribution properties of the plane EUI sources do not follow those of the young pulsar population. We conclude that even though a fraction of the EUI sources may have young pulsar counterparts, the majority of them follow a distribution that is similar to the molecular cloud distribution in nearby spiral arms.
\end{abstract}

Key words. gamma rays: observations - stars: pulsars: general - ISM: clouds

\section{Introduction}

Most of the EGRET unidentified (EUI) high energy gammaray sources detected within a few degrees of the Galactic equatorial plane are considered to be Galactic with the possible exceptions of a few extragalactic sources that could be seen through the dense interstellar medium. The EGRET Third catalog (Hartman et al. 1999) lists 170 sources that are not readily identified with any possible sources at other wavelengths, of which 74 sources are within $\pm 10^{\circ}$ of the Galactic equator. In many cases, the large EGRET positional error box $\left(\sim 1^{\circ}\right.$ or more) has prevented the process of identification with a unique counterpart.

The detection of some radio pulsars such as the Crab and Vela pulsars as well as the detection of the Geminga radio-quiet pulsar in gamma rays have prompted the argument that counterparts for many of the EUI sources could be pulsars (Halpern \& Ruderman 1993; Helfand 1994; Mukherjee et al. 1995; Yadigaroglu \& Romani 1997; Zhang et al. 2000; Harding \& Zhang 2001). Searches undertaken by sensitive $\mathrm{X}$-ray (Chandra) and radio (Parkes Multibeam Survey) telescopes have yielded new pulsar discoveries within EUI error boxes (Halpern et al. 2001; D’Amico et al. 2001). A correlative study of Galactic plane EUI sources and Parkes multibeam

Send offprint requests to: D. Bhattacharya,

e-mail:dipen@tigre.ucr.edu radio survey pulsars has yielded 14 new positional conincidences (Torres et al. 2001). The mid latitude EUI sources that are suggested to be associated with the Gould Belt (Grenier et al. 2000; Gehrels et al. 2000) are also modeled with off-beam gamma-ray pulsars (Harding \& Zhang 2001). Furthermore, a number of EUI sources from the Second EGRET catalog (Thompson et al. 1995) are found to be spatially correlated with OB associations (Kaaret \& Cottam 1996). As pulsars are one of the end products of young OB stars, this correlation, in principle, can be used as a basis for a pulsar-EUI source connection (Zhang \& Cheng 1998).

There is also significant statistical evidence for EUI sources to be supernova remnants (Sturner \& Dermer 1995; Esposito et al. 1996; Romero et al. 1999; Combi et al. 2001) where gamma rays could result from the hadronic interaction of shock accelerated protons and nuclei with the ambient interstellar matter. A few massive Wolf-Rayet and Of stars are also found to be positionally coincident with EUI sources (Kaul \& Mitra 1997; Romero et al. 1999).

It is understood that a single population model is not sufficient to fit all the spatial, spectral and luminosity characteristics of the EUI sources. In this work, we present an analysis of aspects of the global distribution of EUI sources $(>100 \mathrm{MeV})$ near the Galactic plane $\left(|b|<5^{\circ}\right)$ including their $\log N-$ $\log S$ distribution (Sect. 2) and angular correlation function (Sect. 3). An unbiased and complete $\log N-\log S$ relation 
samples the source distribution for a specific source geometry without the knowledge of the source luminosity function or the distance. We discuss the systematic effects that can give rise to errors in the $\log N-\log S$ analysis. Methods of rectifying such systematic effects are also discussed. The angular correlation function offers insight to the global nature of a collection of sources by providing their clustering properties. We compare these EUI source properties directly with pulsar properties to determine the overall nature of the pulsar contribution to EUI sources. The discussion and conclusion of the work are presented in Sect. 4.

\section{2. $\log N-\log S$ analysis}

As an analytical tool for the study of the global properties of the high energy gamma-ray sources, the $\log N-\log S$ graph was used by Bignami \& Caraveo (1980) to derive differences in the longitudinal distribution of COS-B Galactic plane catalog objects. The $\log N-\log S$ approach was later used by Özel \& Thompson (1996) in the analysis of EUI sources. They found that the high latitude EUI sources are consistent with an isotropic extragalactic distribution. These authors also found the sample to be exposure limited and not dominated by background or source confusion. This, however, is not applicable to the Galactic plane EUI sources due to the difficulties in appraising the source definition within the diffuse emission. A comprehensive $\log N$ - $\log S$ study was carried out by Reimer \& Thompson (2001) who concluded that the existing models explaining the spatial arrangements of the EUI sources do not match the observed $\log N-\log S$ distribution.

The $\log N-\log S$ curve for the Galactic plane EUI sources given in the Third EGRET Catalog should represent the disk distribution of these sources with respect to the Sun. From the center of a simple uniform thin disk distribution of sources with constant luminosity, one expects to detect $N$ sources above the flux level $S$ according to $N \sim S^{-\beta}$, where $\beta=1$. If $\beta$ is greater than 1 , the source density increases as a function of the distance from the observer, whereas a decrease in the source density as a function of distance will result in $\beta$ less than 1 . However, when the observer is off-center (as in the case of the Sun), the integral source counts are represented by two power laws:

$$
\begin{gathered}
N(S) \sim S^{-\beta_{1}}, S>S_{\mathrm{c}} \\
N(S) \sim S^{-\beta_{2}}, S<S_{\mathrm{c}} .
\end{gathered}
$$

The break at a flux value of $S_{\mathrm{c}}$ is dictated by the position of the observer with respect to the center. For a zero-width disk with a radius of 16 unit lengths and the observer at 8 unit lengths from the center, $\beta_{1} \sim 1$ and $\beta_{2} \sim 0.6$ where we assume a uniform source distribution, constant source luminosity, no extinction in the interstellar medium and infinite detector sensitivity. The break occurs at $\sim 8$ unit lengths from the observer.

Both the radio pulsars and known gamma-ray pulsars have luminosity distributions covering several orders of magnitude. In a simulation where the EUI source luminosities are assumed to be distributed normally, we find $\beta_{1}$ remains close to 1 as we vary $\sigma$ of the luminosity distribution. The corresponding $\beta_{1}$ for $\sigma /$ (Peak Luminosity) ratios of $0.05,0.4,1.0$ and 2.0 are 1.1 , $1.09,1.07$, and 1.04 , respectively. The slope becomes slightly steeper as the value of $\sigma$ is decreased.

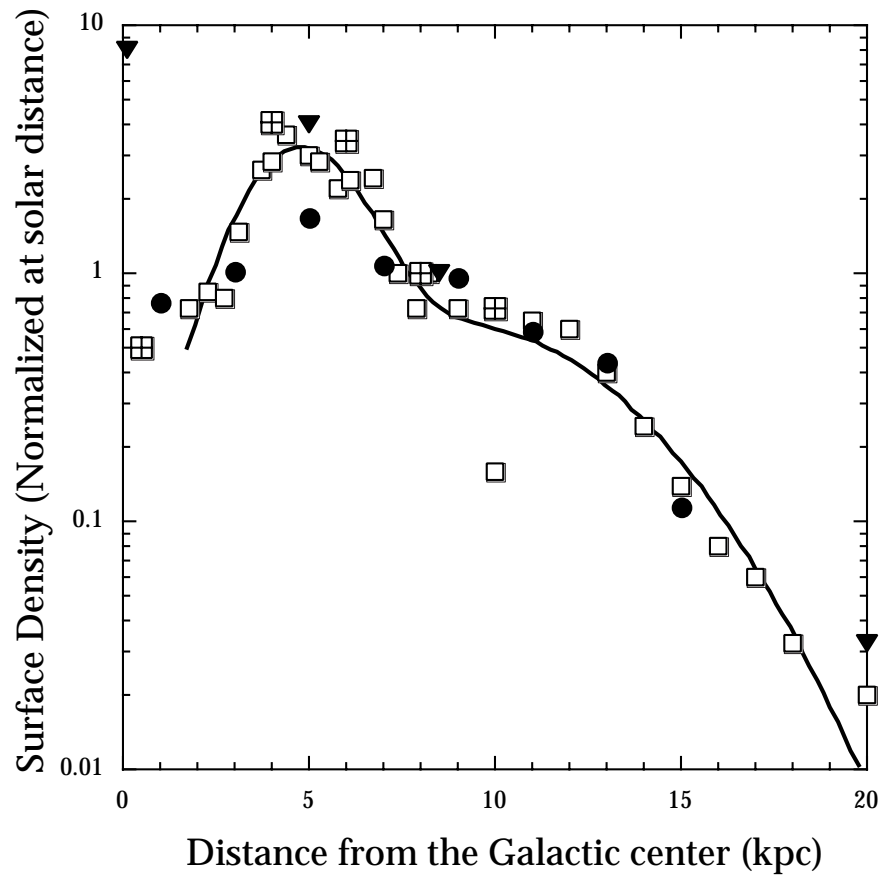

Fig. 1. The distribution of molecular clouds (open square), OB associations (crossed square), supernova remnants (filled circle) and stars (inverted filled triangle) as functions of Galactic radius. A functional fit to the molecular gas distribution is also shown.

We follow other works to assume that the EUI sources are associated with star forming regions, OB associations, supernova remnants (SNR) and/or pulsars. In Fig. 1, we show the surface density distribution of molecular clouds (Wiliams \& McKee 1997), OB associations (McKee \& Williams 1997), SNR (Case \& Bhattacharya 1998) and stars (Lacey \& Fall 1985) as functions of Galactocentric radius. The radial distribution of molecular gas in the Galaxy is given by Williams $\&$ McKee (1997) using the data of Bronfman et al. (1988) and Wouterloot et al. (1990). We use a functional fit to the two-dimensional distribution of the molecular gas as our distribution function for sources in the Galactic plane. Using this smooth source distribution and assuming a constant luminosity for these sources we simulated a $\log N-\log S$ relation that is shown in Fig. 2. The relation can be approximated by $N(S) \sim S^{-1.1}$ within $8 \mathrm{kpc}$ of the Sun which is expected from a flat disk source distribution. If EUI source generation is related to star forming regions we would expect a similar $\log N-\log S$ for them.

\subsection{Systematic effects}

There are several systematic effects that can introduce errors to the $\log N-\log S$ curve (Hasinger et al. 1993). These include i) the merging of two or more sub-threshold sources present in the same resolution point spread function (PSF) to produce a single detected source, ii) the merging of two or more abovethreshold sources present within the instrument resolution into a single bright source, and iii) the difficulty in defining discrete sources within the strong diffuse continuum. To correctly interpret the unidentified $\log N-\log S$ curve we need to take into 


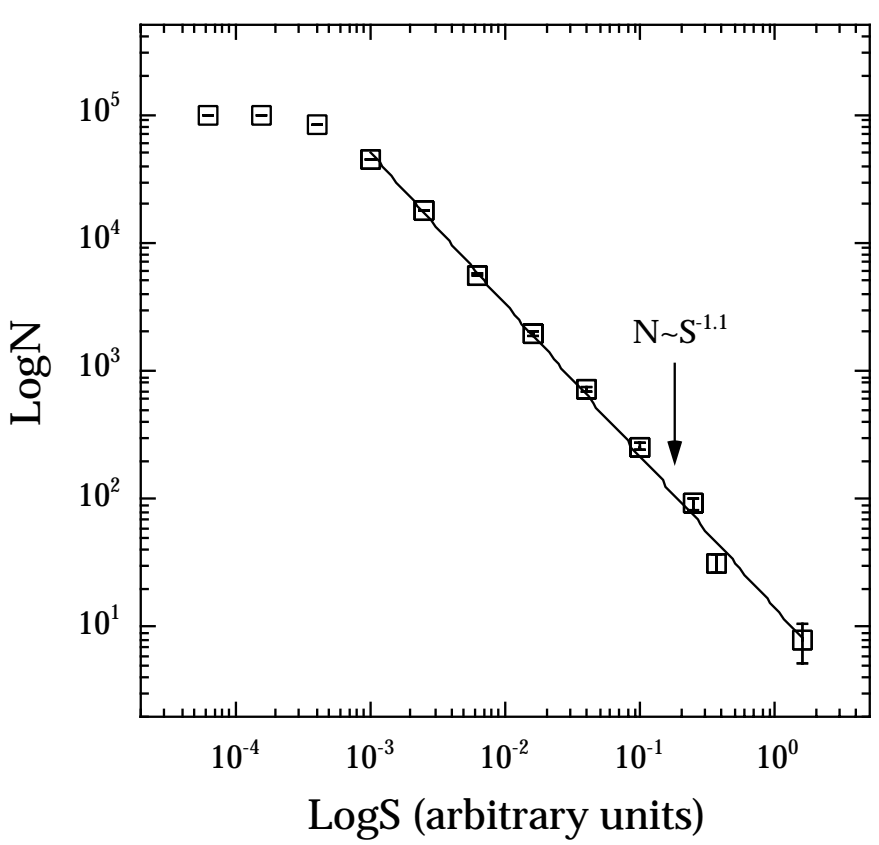

Fig. 2. A simulated $\log N-\log S$ relation assuming a source distribution according to the smooth molecular gas distribution and a constant source luminosity.

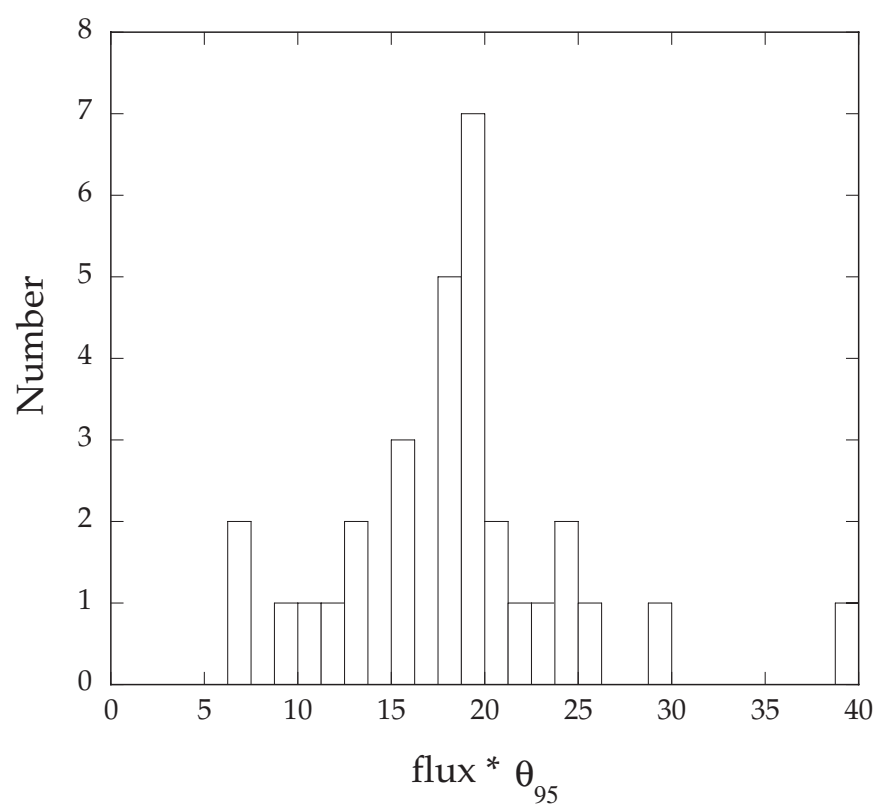

Fig. 3. Flux $\times \Theta_{95}$ distribution for EUI sources. The flux values are in units of $10^{-8} \mathrm{ph} \mathrm{cm}^{-2} \mathrm{~s}^{-1}$.

account both the position and flux uncertainties of the detection. However, both these parameters are very difficult to determine as most of the low latitude EUI sources lie in regions of intense diffuse emission.

It can be argued that for the first two effects, either the flux or the PSF $\left(\Theta_{95}\right.$ of the EGRET Third Catalog) value will be higher than the average so that a simple product of these two parameters (Flux $\left.\times \Theta_{95}\right)$ for a particular source would be high if it is in a confused region. We have plotted the $\left(\right.$ Flux $\left.\times \Theta_{95}\right)$ for all Galactic plane EUI sources $\left(>100 \mathrm{MeV},|b|<5^{\circ}\right)$ in Fig. 3.
Here sources with high $\left(\right.$ Flux $\left.\times \Theta_{95}\right)$ values would represent confused detections. Indeed, the 3 sources (3EG J1903+0550, 3EG J1639-4702 and 3EG J1655-4554) with (Flux $\left.\times \Theta_{95}\right)>25$ are termed as either extended or confused in Third EGRET catalog. However, for lower-significance sources, the $95 \%$ contour can be extremely irregular, or sometimes even not closed. The Third Catalog lists the error circle for those sources as $1.62 \times$ the radius of the $68 \%$ contour. We find only one source (3EG J1027-5817) falling into this category (which has the fourth highest (Flux $\times \Theta_{95}$ ) of 24.4). Including the latter, we have decided to discard the four sources with highest $\left(\right.$ Flux $\left.\times \Theta_{95}\right)$ in the $\log N-\log S$ analysis.

However, this approach does not solve the completeness problem of the sample. The completeness of the EGRET sources has been investigated by Hartman et al. (1999). Reimer (2001) also discusses the various detectability issues associated with EUI source distribution with respect to exposure and diffuse background. The detectability of a source at a position $(l, b)$ is proportional to the square root of the ratio of the EGRET exposure to the intensity of the diffuse gammaray emission at that point. This definition was used by Reimer (2001) to produce an EGRET detectability map. Following Mattox et al. (1996), we consider the source detectability, $d$, for a specific observation with exposure $e$ and diffuse background, $b$, to be given by

$d(f, l, b) \sim f \sqrt{\frac{e}{b}}$

where $f$ is the source flux. To account for the non-uniform detectability for point sources, the $\log N-\log S$ curve has to be normalized using the detectability function, $d(f, l, b)$. This can be done by replacing unity for each source at a given $(l, b)$ by $1 / d(f, l, b)$.

Following Gehrels et al. (2000) we assume those EUI sources to be "steady" (see also Carraminana 2001; Grenier 2001) for which the most significant EGRET detection is obtained using the cumulative data from April 1991 to October 1995 (P1234 in the Third EGRET Catalog). This benchmark allows us to address the problems associated with the EUI source variability. Tompkins (1999) calculates a $\tau$ variability parameter using a Likelihood approach to EGRET data and identifies sources to be highly variable if their $68 \%$ lower limit of $\tau$ happens to be above 1 . We find all P1234 sources, except for 3EG J1655-4554, have a $\tau 68 \%$ lower limit of less than 0.5 . This source happens to have the third highest (Flux $\times$ $\left.\Theta_{95}\right)$ value and has already been excluded. Furthermore, the Third Catalog suggests 3EG J0628+1847 could be an artifact due to its proximity to the Geminga pulsar and we exclude this source from our analysis. By choosing sources within $\pm 5^{\circ}$, we also eliminate the possible confusion with Gould Belt sources. Two P1234 sources, 3EG J0824-4610 and 3EG J0827-4247, which are thought to be artifacts associated with the Vela region (Hartman et al. 1999), are also excluded. By excluding the 7 sources described above, the total number of EUI sources within $|b|<5^{\circ}$ is 26 . The cumulative number of sources are then calculated per logarithmic flux intervals. The resulting $\log N-\log S$ curve, normalized for the source detectability 


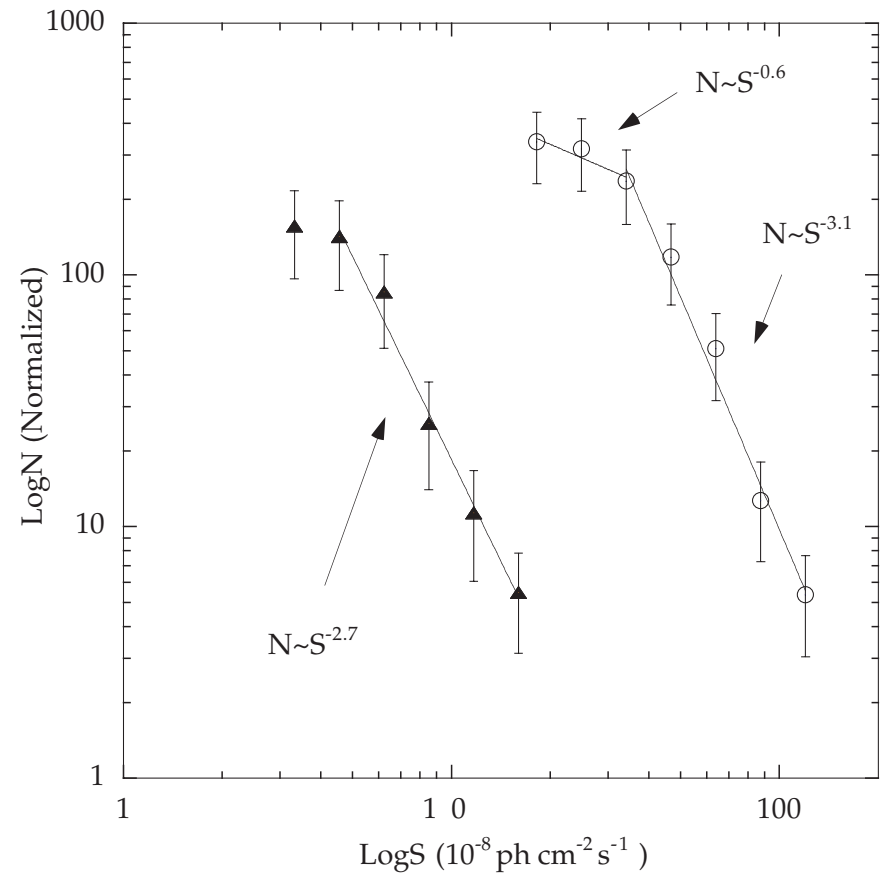

Fig. 4. The $\log N-\log S$ curve of EUI sources with $|b|<5^{\circ}$ (open circles). The $\log N-\log S$ plot for EUI sources with $\mathrm{GeV}$ emission is also shown (triangles).

function, is shown in Fig. 4. The power law fit to the $\log N-$ $\log S$ curve is given below.

$N(S) \sim S^{-3.1 \pm 0.4}$

The figure shows the break in the curve that occurs at about $3.4 \times 10^{-7} \mathrm{ph} \mathrm{cm}^{-2} \mathrm{~s}^{-1}$. The steep power law index could be indicative of i) a nearby source concentration that increases with distance from us, ii) a non-uniform distribution or iii) could be the result of other selection effects. The steep slope value is similar to that derived by Gehrels et al. (2000) and Reimer \& Thompson (2001).

On the other hand, EUI sources, detected above $1 \mathrm{GeV}$, are considered relatively confusion free due to their smaller PSF (Thompson et al. 1993) and can be used to construct the $\log N-$ $\log S$ plot. The GeV sources are selected from the GeV source catalog of Lamb \& Macomb (1997), which is derived solely from the above $1 \mathrm{GeV}$ count maps. However, we used flux values obtained by Roberts et al. (2001), who rederived the above $1 \mathrm{GeV}$ fluxes for most of the sources by including all nearby $3 \sigma$ sources in the fit. A total of 16 sources with $|b|<5^{\circ}$ are used. We have discarded GeV 1825-1319 because it did not satisfy the P1234 flux criterion. All known pulsars are also not included. We have normalized the GeV sources for flux, exposure and background as we have done before for fluxes above $100 \mathrm{MeV}$. We show this curve in Fig. 4; the weighted fit can be described as $N(S) \sim S^{-2.7 \pm 0.4}$. The GeV sources represent a subset of the Galactic plane EUI sources and might represent a separate population altogether. However, the steepness of their $\log N$-Log $S$ curve also seems to indicate their non-uniform distribution in the disk.

\section{2. $\log N-\log S$ analysis of pulsars}

The pulsar fluxes and ages are taken from the Australia Telescope National Facility (ATNF) Pulsar Catalog ${ }^{1}$, which currently lists 1395 radio pulsars. ATNF is a compilation catalog of known pulsar databases given by Taylor et al. (1993), the Parkes Multibeam (PMB) Pulsar Survey (Manchester et al. 2001) and the Swinburne Intermediate Latitude Pulsar Survey (Edwards et al. 2001). The two latter surveys are both made using the ATNF Parkes 64-m radio telescope.

The most important selection effects in the observed pulsar sample include the flux inverse square law, pulse dispersion and scattering. The low frequency observation provides a better pulsar spectra, wheareas the high frequency observation is favored against dispersion smearing, scattering and background galactic noise. The differences in the arrival times at different frequencies within an observation band can smear out pulse signals, especially signals from millisecond pulsars, and render them undetectable (Nice 1995). Dispersion and scattering are severe for distant pulsars where the number of free electrons in the line of sight become large. A technique to correct for the selection effects is to make use of a scale factor that determines the level of under-sampling of pulsar detection in a certain Galactic volume (Phinney \& Blandford 1981; Vivekanand \& Narayan 1981). However, under-sampling is less pronounced within a few kpc of the sun and the dispersion/scattering effects are considerably less for surveys at observing frequencies of $\geq 1400 \mathrm{MHz}$ (Clifton et al. 1992).

By selecting only those pulsars that fall within the sensitivity range of EGRET, we can restrict the range of dispersion measures. This is achieved by assuming that if EUI sources are indeed pulsars, their luminosity is approximately equal to the Crab pulsar luminosity, but with a beaming angle of $4 \pi$. As the Crab pulsar remains the most luminous of all detected gamma-ray pulsars this naive argument - at worst over estimates the standard luminosity and extends the range of EGRET detectability. Employing this restriction, we find the average dispersion measure (DM) for the selected pulsars to be approximately $200 \mathrm{pc} \mathrm{cm}^{-3}$. Towards the Galactic center this corresponds approximately to a distance of $3.63 \mathrm{kpc}$ and an estimated scattering timescale of $0.271 \mathrm{~ms}$ at $1 \mathrm{GHz}$. The $\log N-\log S$ slope of these selected pulsars detected in $400 \mathrm{MHz}$ (194 total) and $1400 \mathrm{MHz}$ (366 total) are shown in Figs. 5 and 6, respectively. Both the plots can be approximated by $N \sim S^{-1.0}$. The young pulsars, with ages less than a million years, which are considered potential gamma-ray emitters, have slopes similar to the total pulsar $\log N-\log S$ curves. These results suggest that the general pulsar population follows a Galactic disk distribution. The similarity of the two $\log N$ $\log S$ slopes for 400 and $1400 \mathrm{MHz}$ indicates that the selection effects are not dominant in our restricted pulsar samples.

However, to verify these results we performed another set of $\log N-\log S$ analysis where we artificially restricted the pulsar distances to $3 \mathrm{kpc}$. This translates to a mean DM of $125 \mathrm{pccm}^{-3}$ and a scattering timescale of $0.098 \mathrm{~ms}$ at $1 \mathrm{GHz}$. In this case, the $\beta$ s for the total pulsar $\log N-\log S$

${ }^{1}$ http://www.atnf.csiro.au/research/pulsar/catalogue 


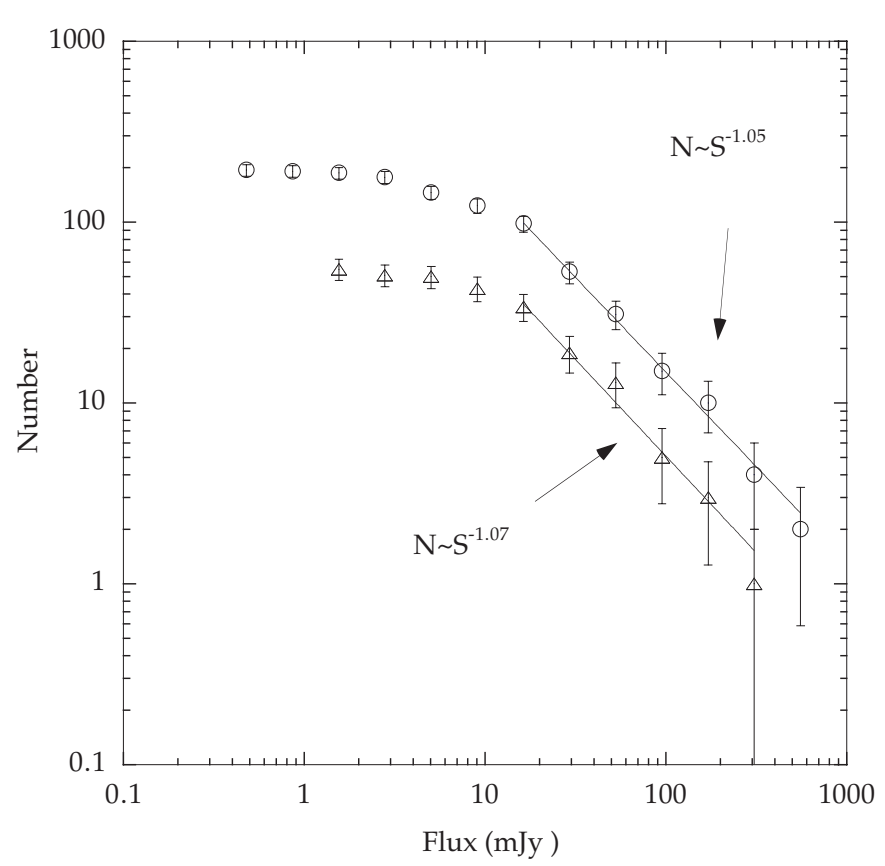

Fig. 5. The $\log N-\log S$ plot of the Galactic pulsars detected at $400 \mathrm{MHz}$ within $|b|<5^{\circ}$. The $\log N-\log S$ of pulsars with age $<10^{6}$ years is also shown (triangles).

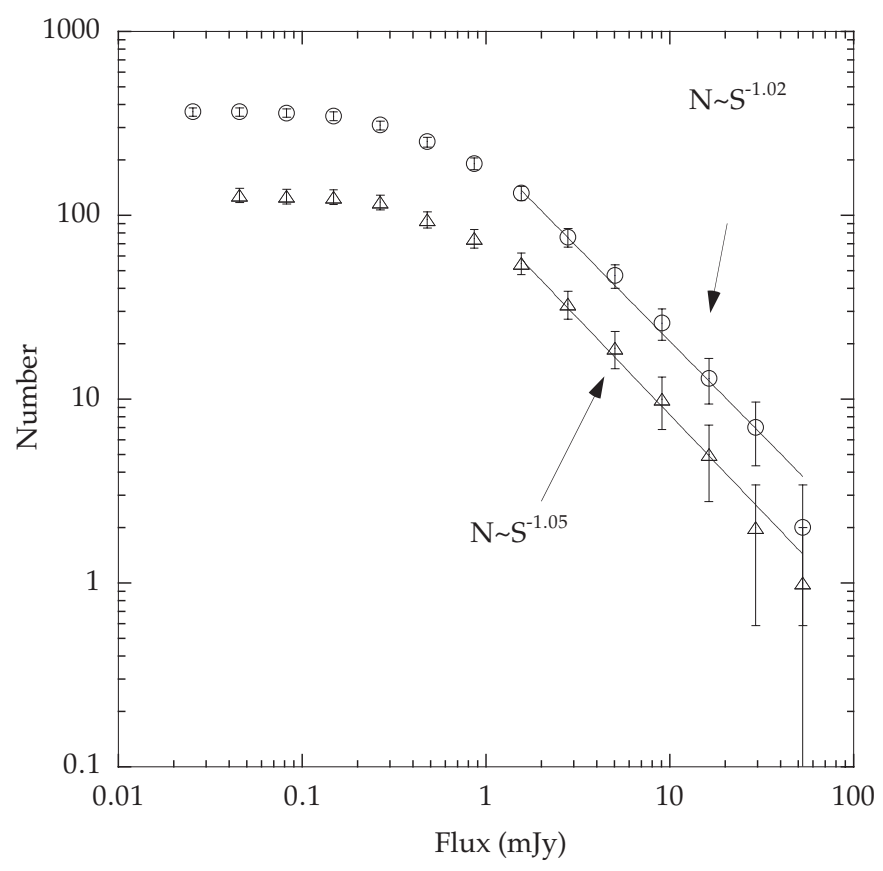

Fig. 6. The $\log N-\log S$ plot of the Galactic pulsars detected at $1400 \mathrm{MHz}$ within $|b|<5^{\circ}$. The $\log N-\log S$ of young pulsars with age less than $10^{6}$ years is also shown (triangles).

are $0.88 \pm 0.12$ and $0.84 \pm 0.09$ at 400 and $1400 \mathrm{MHz}$, respectively. The corresponding $\beta$ s for young pulsars are $1.22 \pm 0.42$ and $0.76 \pm 0.23$. These results indicate that the nearby pulsars also follow a disk distribution.

\section{Angular correlation}

The distribution of the EUI sources in the Galactic plane shows signs of clustering in several regions. The clustered regions include, besides the direction towards the Galactic center, the Cygnus and Carina OB associations. To quantify the scales of such clustering a two-point angular correlation function can be helpful. The correlation function for EUI sources can be directly compared with the pulsar correlation function to establish whether these two populations share the same clustering property. This function, $w(\theta)$, is needed to determine the probability $\delta P(\theta)$ of finding two objects separated by an angle $\theta$ and located within two small solid angles $\delta \Omega_{1}$ and $\delta \Omega_{2}$ (Peebles 1973), where $\delta P(\vartheta)=n^{2}[1+w(\theta)] \delta \Omega_{1} \delta \Omega_{2}$ and $n$ is the mean number density of the sources. When $w(\theta)=0$, the sources are assumed to be not clustered, whereas positive and negative $w(\theta)$ 's denote clustering and anti-clustering behaviors, respectively. An estimation of the correlation function can be constructed by directly comparing the pairings of the detected sources to that of a random sample. If $N_{\mathrm{d}}$ and $N_{\mathrm{r}}$ are the numbers of observed sources and the random sources, then we can use the following definition of the angular correlation function:

$w(\theta)=\left[\frac{2 N_{\mathrm{r}}}{\left(N_{\mathrm{d}}-1\right)}\right] \frac{N_{\mathrm{dd}}}{N_{\mathrm{dr}}}-1$

where $N_{\mathrm{dd}}$ is the number of EUI source pairs and $N_{\mathrm{dr}}$ is the number of EUI source and random source pairs. The term within the square bracket is a normalization factor. The error for $w(\theta)$ is given by (Peebles 1973):

$\sigma_{w}=\sqrt{\left[\frac{2 N_{\mathrm{r}}}{\left(N_{\mathrm{d}}-1\right)}\right]\left(\frac{1+w(\theta)}{N_{\mathrm{dr}}}\right)}$.

Since, we intend to find the clustering scale only for the detected EUI sources, the normalization method used for the $\log N-\log S$ analysis is not applicable for the angular correlation analysis. For this analysis, we generate a uniform random sample 10 times larger than the number of EUI sources for $|b|<$ $5^{\circ} ; w(\theta)$ and $\sigma_{w}$ are also calculated for this sample. This process is repeated 100 times and an average $w(\theta)$ and $\sigma_{w}$ are determined. Evaluations of correlation functions are also carried out for the pulsars in the ATNF catalog with $|b|<5^{\circ}$. Pulsars situated only between the longitude range $260^{\circ}$ and $50^{\circ}$ are selected so that a correlation function can be established within a contiguous segment of the sky. This range is selected to match PMB surveys with other telescope observations. Pulsars with undetermined ages are not included. In Fig. 7, we present the correlation function for the 26 EUI sources. We have used logarithmic bins between $1^{\circ}$ and $20^{\circ}$ with a bin of $\Delta \log \theta=1.28$. The clustering properties of the EUI sources below $1^{\circ}$ could not be ascertained due to the limiting resolution of EGRET. The upper limit is bound only by Galactic structures which are assumed to be less than $20^{\circ}$. The peak value of the angular correlation function at $3^{\circ}$ has a significance of only $2.5 \sigma$. Hence, we conclude that no significant clustering scale of EUI sources has been found by this analysis.

In Figs. 8 and 9, we show the two point correlation functions obtained for the young and old pulsar populations. The lower limit is constrained to $0.5^{\circ}$ due to sparse statistics. Pulsars falling within the EGRET gamma-ray detectable range are included and a total of 124 young pulsars $\left(t_{\text {age }}<10^{6} \mathrm{yrs}\right)$ 


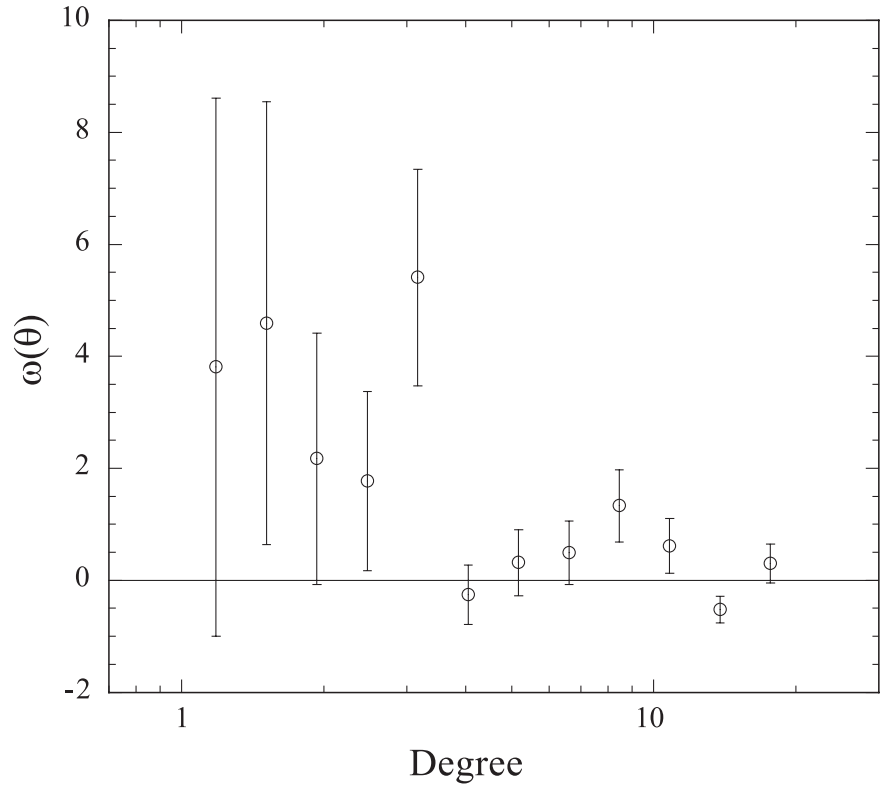

Fig. 7. The angular correlation function of EUI sources within $|b|<5^{\circ}$.

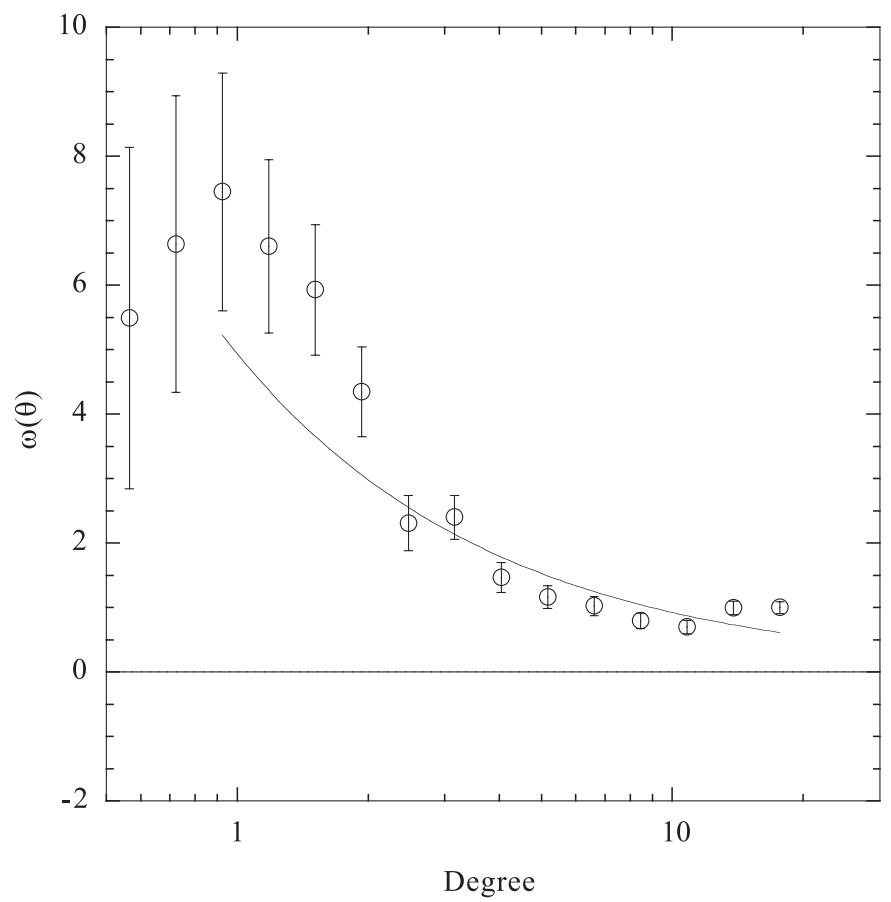

Fig. 8. The angular correlation function of Galactic pulsars with ages less than $10^{6}$ years.

and 281 old pulsars $\left(t_{\text {age }}>10^{6} \mathrm{yrs}\right)$ are used for this analysis. Here we have also used pulsars that have zero flux values in the Catalog. The correlation function for young pulsars peaks at $\theta \sim 1^{\circ}$ (with a significance of $4 \sigma$ ) and then follows a power law decay which can be approximated by the form $\theta^{-\alpha}$ where $\alpha$ is 0.7 . The correlation function for the old pulsars follows a power law decay with an index of 0.5 , without exhibiting a peak. This is consistent with the fact that older pulsars would disperse from the regions of their birth thereby losing their clustering properties.

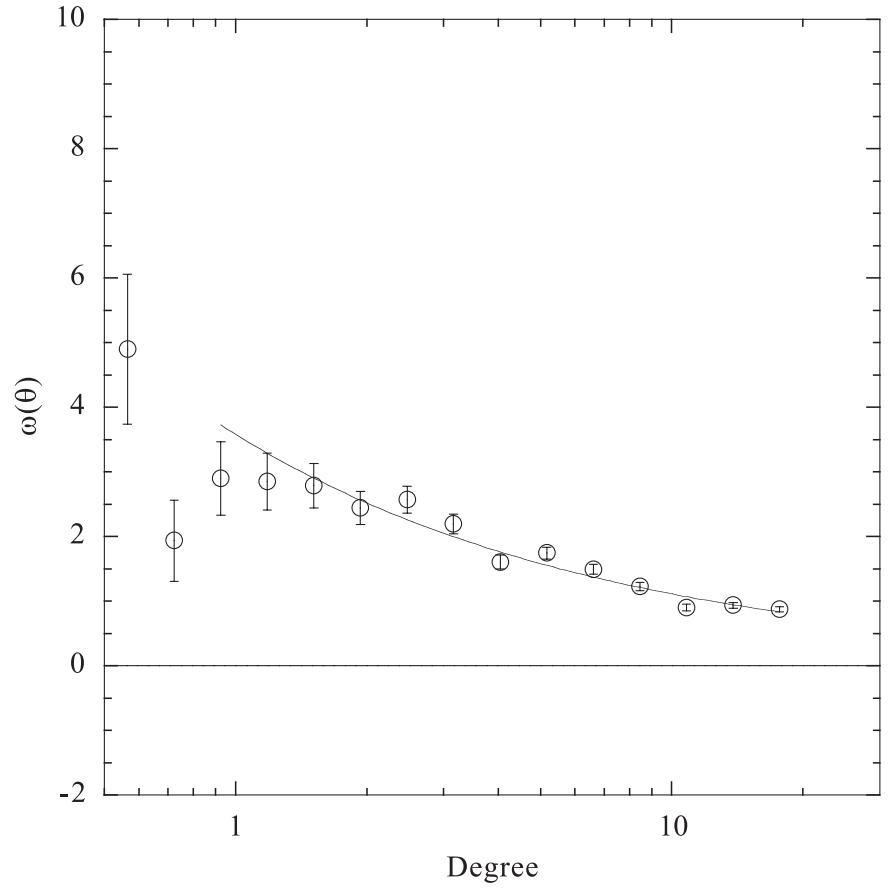

Fig. 9. The angular correlation function of Galactic pulsars with ages greater than $10^{6}$ years.

Although it would be tempting to suggest that these correlation results point to different origins of pulsars and EUI sources, the small number of EUI sources used in this analysis warrants more EUI source detections to establish a firm clustering scale. Future detections of EUI sources by GLAST possibly will resolve the issue.

\section{Discussion and conclusion}

We used the $\log N-\log S$ graph of the EUI sources to understand their inherent geometrical distribution. If these sources are associated with a population which is uniformly distributed in a Galactic plane, we would expect a $N \sim S^{-1}$ relation from the $\log N-\log S$ curve. However, the bright EUI sources with flux values greater than $3.4 \times 10^{-7} \mathrm{ph} \mathrm{cm}^{-2} \mathrm{~s}^{-1}$ have a power law slope of approximately -3 . This steep index can be explained by an increase in the source population density away from us. The concentrated sources in the nearby spiral arms can simulate such a distribution.

To explore the above idea we studied the distribution of molecular clouds in the Carina and Perseus arms. The infrared $\log N-\log S$ curves of the compact molecular clouds (CMC) in the first and fourth quadrants have slopes ranging from 1.65 to 2.25 (Little \& Richards 1989). If molecular clouds are associated with EUI sources, we might derive a phenomenological $\log N-\log S$ curve based on the molecular cloud masses. In Fig. 10, we present such a $\log N-\log S$ plot for gammaray sources that might be associated with the Carina arm giant molecular clouds. The molecular cloud data have been taken from Grabelsky et al.'s (1988) Columbia CO survey of the Southern Galactic Plane. In our analysis, the gamma-ray source number is assumed to be proportional to the molecular cloud mass. Assuming a luminosity of $2 \times 10^{35} \mathrm{ergs} \mathrm{s}^{-1}$ for the 


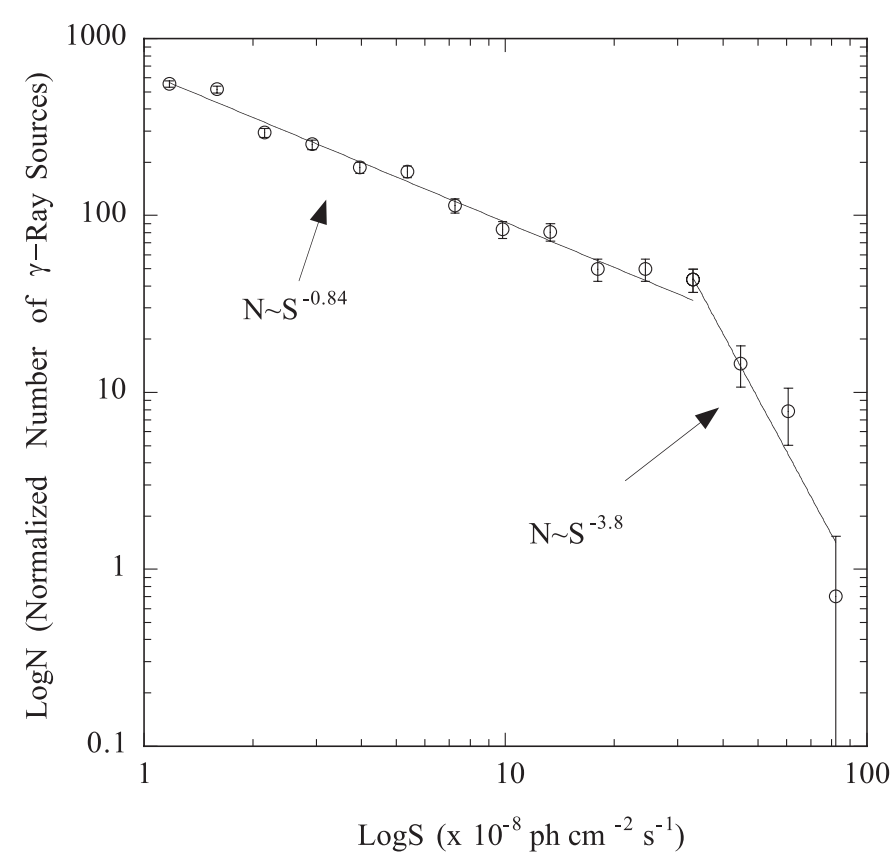

Fig. 10. A simulated $\log N-\log S$ plot for gamma-ray sources assuming they are associated with the molecular clouds in the Carina arm.

gamma-ray sources, we find a $\log N-\log S$ slope of -3.8 . This luminosity is chosen so that the break of the EUI $\log N-\log S$ curve occurs at about $3.4 \times 10^{-7} \mathrm{ph} \mathrm{cm}^{-2} \mathrm{~s}^{-1}$. This luminosity is also within the range given by Kanbach et al. (1996) for EGRET Galactic plane sources. The steep slope is indicative of the molecular cloud concentration in the spiral arm. Similarly the steepness of the EUI source $\log N-\log S$ could be explained by the concentration of those sources in the spiral arms. The break in Fig. 10 corresponds to a distance of $3 \mathrm{kpc}$ (from the distances given for the clouds). It would imply that the observed EUI source distribution is within a few kpc. For clouds that are located in the Perseus arm, which is outside the solar circle, the $\log N-\log S$ curve has a slope value of $\sim-2.8$. The Perseus molecular cloud data have been taken from Leisawitz (1990).

The $\log N-\log S$ curves of the radio pulsars, both young and old, can be fit with power laws that have indices close to -1.0 . This would seem to indicate that the pulsar population is uniformly distributed in the Galactic thin disk. We also find there is no clear difference in the distribution of pulsars throughout the Galaxy and the pulsars only within the EGRET detection range. The $\log N-\log S$ graph for the EUI sources with high flux levels produce a steep curve which is not indicative of a uniform disk population (e.g., pulsar), but rather of the molecular cloud distribution in spiral arms within the solar circle up to an approximate distance of $3 \mathrm{kpc}$.

The angular correlation analysis failed to establish a clustering scale for the detected EUI sources, whereas the same analysis showed $\mathrm{a} \sim 1^{\circ}$ clustering scale for young pulsars. In view of both the $\log N-\log S$ and angular correlation analyses, it seems that the global distribution properties of the EUI sources do not match that of the young pulsar population. Even though the young pulsars might constitute a fraction of the EUI sources, they are possibly not associated with the majority of the EUI sources. Further observations by future experiments such as GLAST will provide relatively more confusion-free sources; better sensitivity will also result in lower selection effects. The results of this work can be tested with the GLAST data set.

Acknowledgements. We would like to thank D. J. Thompson, D. Dixon and the referee M. Roberts for important suggestions. This work was supported in part by NASA through grant NAG5-5116.

\section{References}

Bignami, G. F., \& Caraveo, P. A. 1980, ApJ, 1161

Bronfman, L., Cohen, R. S., Alvarez, H., et al. 1988, ApJ, 324, 248

Carraminana, A. 2001, in The Nature of Unidentified Galactic High-Energy Gamma-Ray Sources, Puebla, Mexico, ed. A. Carraminana, O. Reimer, \& D. J. Thompson (Dordrecht: Kluwer Academy Publishers), 107

Case, G., \& Bhattacharya, D. 1998, ApJ, 504, 761

Clifton, T. R., Lyne, A. G., Jones, A. W., et al. 1992, MNRAS, 254, 177

Combi, J. A., Romero, G. E., Benaglia, P., et al. 2001, A\&A, 366, 1047

D’Amico, N., Kaspi, V. M., Manchester, R. N., et al. 2001, ApJ, 552, L45

Edwards, R. T., Bailes, M., van Straten, W., et al. 2001, MNRAS, 326, 358

Esposito, J. A., Hunter, S. D., Kanbach, G., et al. 1996, ApJ, 461, 820

Grabelsky, D. A., Cohen, R. S., Bronfman, L., et al. 1988, ApJ, 331, 181

Gehrels, N., Macomb, D. J., Bertsch, D. L., et al. 2000, Nature, 404, 363

Grenier, I. A. 2000, A\&A, 364, L93

Grenier, I. A. 2001, in The Nature of Unidentified Galactic GammaRay Sources, Puebla, Mexico, ed. A. Carraminana, O. Reimer, \& D. J. Thompson (Dordrecht: Kluwer Academic Publishers), 51

Halpern, J. P., \& Ruderman, M. A. 1993, ApJ, 415, 286

Halpern, J. P., Camilo, F., Gotthelf, E. V., et al. 2001, ApJ, 552, 125

Harding, A. K., \& Zhang, B. 2001, ApJ, 548, L37

Hartman, R. C., Bertsch, D. L., Bloom, S. D., et al. 1999, ApJS, 123, 79

Hasinger, G., Burg, R., Giacconi, R., et al. 1993, A\&A, 275, 1

Helfand, D. J. 1994, MNRAS, 267, 490

Kaaret, P., \& Cottam, J. 1996, ApJ, 462, L35

Kanbach, G., Bertsch, D. L., Dingus, B. L., et al. 1996, A\&AS, 120, 461

Kaul, R. K., \& Mitra, A. K. 1997, Proc. of the Fourth Compton Symp., ed. C. D. Dermer, M. S. Strickman, \& J. D. Kurfess (New York: AIP), 1271

Lacey, C. G., \& Fall, S. M. 1985, ApJ, 290, 154

Lamb, R. C., \& Macomb, D. J. 1997, ApJ, 488, 872

Leisawitz, D. 1990, ApJ, 359, 319

Little, L. T., \& Richards, P. J. 1989, MNRAS, 240, 397

Manchester, R. N., Lyne, A. G., Camilo, F., et al. 2001, MNRAS, 328, 17

Mattox, J. R., Bertsch, D. L., Chiang, J., et al. 1996, ApJ, 461, 396

McKee, C. F., \& Williams, J. P. 1997, ApJ, 476, 144

Mukherjee, R., Bertsch, D. L., Dingus, B. L., et al. 1995, ApJ, 441, L61

Nice, D. J. 1995, in The Lives of the Neutron Stars, Turkey, ed. M. A. Alpar, U. Kiziloglu, \& J. van Paradijs (Dordrecht: Kluwer Academic), 225

Özel, M. E., \& Thompson, D. J. 1996, ApJ, 463, 105 
Parkes Multibeam Pulsar Survey 2001, http://www .atnf.csiro.au/research/pulsar/psr Peebles, P. J. E. 1973, ApJ, 185, 413

Phinney, E. S., \& Blandford, R. D. 1981, MNRAS, 194, 137

Reimer, O. 2001, in The Nature of Unidentified Galactic High-Energy Gamma Ray Sources, ed. A. Carraminana, O. Reimer, \& D. J. Thompson (Netherlands: Kluwer Academic Publishers), 17

Reimer, O., \& Thompson, D. J. 2001, 27th International Cosmic Ray Conference, 7-15 August 2001 (Hamburg, Germany)

Roberts, M. S. E., Romani, R. W. \& Kawai, N. 2001, ApJS, 133, 451

Romero, G. E., Benaglia, P., \& Torres, D. F. 1999, A\&A, 348, 868

Sturner, S. J., \& Dermer, C. D. 1995, A\&A, 293, L17

Taylor, J. H., Manchester, R. N., \& Lyne, A. G. 1993, ApJS, 88, 529
Thompson, D. J., Bertsch, D. L., Fichtel, C. E., et al. 1993, ApJS, 86, 629

Thompson, D. J., Bertsch, D. L., Dingus, B. L, et al. 1995, ApJS, 101, 259

Tompkins, W. 1999, Ph.D. Thesis

Torres, D. F., Butt, Y. M., \& Camilo, F. 2001, ApJ, 560, L155

Vivekanand, M., \& Narayan, R. 1981, JApA, 2, 315

Williams, J. P., \& McKee, C. F. 1997, ApJ, 476, 166

Wouterloot, J. G. A., Brand, J., Burton, W. B., et al. 1990, A\&A, 230, 21

Yadigaroglu, I.-A., \& Romani, R. W. 1997, ApJ, 476, 347

Zhang, L., \& Cheng, K. S. 1998, A\&A, 335, 234

Zhang, L., Zhang, Y. J., \& Cheng, K. S. 2000, A\&A, 357, 957 